\title{
Détourner des publicités, pourquoi ? Pour quoi faire?
}

Thérèse Brouat

\section{(2) OpenEdition \\ Journals}

Édition électronique

URL : http://journals.openedition.org/asp/4390

DOI : 10.4000/asp.4390

ISSN : 2108-6354

Éditeur

Groupe d'étude et de recherche en anglais de spécialité

Édition imprimée

Date de publication : 1 mars 1993

Pagination : 411-435

ISSN : 1246-8185

Référence électronique

Thérèse Brouat, «Détourner des publicités, pourquoi ? Pour quoi faire ? », ASp [En ligne], 1 | 1993, mis en ligne le 13 mai 2014, consulté le 01 mai 2019. URL : http://journals.openedition.org/asp/4390 ; DOI : $10.4000 /$ asp.4390

Ce document a été généré automatiquement le 1 mai 2019.

Tous droits réservés 


\title{
Détourner des publicités, pourquoi? Pour quoi faire?
}

\author{
Thérèse Brouat
}

1 Le détournement fait partie de notre pratique quotidienne d'enseignants de langue. En effet, dès lors que nous utilisons des documents authentiques pour un cours, nous nous servons de textes qui ont été produits par un énonciateur donné, pour un destinataire donné, dans un but spécifique. Or, nous prenons ce moyen de communication qu'est le document, nous le coupons de son origine énonciative et nous l'insérons dans un nouveau contexte énonciatif, celui du cours de langue. Par ailleurs, nous lui assignons des objectifs didactiques étrangers aux objectifs pour lesquels il a été conçu à l'origine.

2 Il s'agit donc bien de détournement puisqu'il y a utilisation d'un support pour une destination qui n'avait pas été prévue. Sans doute s'agit-il d'un détournement légitime, encore faut-il avoir conscience que cette réorientation implique recadrages, mises en relief, fléchages nouveaux, et qu'ainsi, même si l'on utilise des documents authentiques, l'usage que l'on en fait est parfaitement «non naturel». Cette déviation impose à l'enseignant une définition claire de ses objectifs et des moyens à mettre en œuvre pour les atteindre. C'est en effet à un véritable recyclage de matériau que nous nous adonnons.

Pourquoi chercher à détourner des publicités informatiques à l'intention d'étudiants de DUT Informatique? Ces raisons sont de deux ordres, l'une ayant trait au public étudiant qui est le nôtre, l'autre à la nature et à la spécificité du document publicitaire.

À quel public s'adresse un enseignant de département informatique d'IUT ?

Rappelons tout d'abord que l'IUT a pour mission de former des techniciens supérieurs à un niveau Bac + 2. Il s'agit donc d'une formation supérieure courte, destinée normalement à permettre en 2 ans à des bacheliers d'être opérationnels sur le marché du travail. Nous recrutons majoritairement des jeunes ayant un Bac $\mathrm{C}$ ou $\mathrm{D}$, quelques bacs $\mathrm{B}, \mathrm{E}, \mathrm{G}$, mais aussi des jeunes issus de bacs professionnels ainsi que des adultes en formation continue dont le passé scolaire est parfois loin, ce qui suppose de leur part comme de la nôtre une nécessaire adaptation. 
6 Public aux facettes multiples donc, dont le niveau en anglais est également hétérogène. La formation en anglais qui leur est proposée comporte deux parties : une partie d'« anglais général » et une partie d'" anglais informatique ».

7 Or il est bien évident qu'à leur arrivée à l'IUT ces étudiants ne sont pas des spécialistes d'informatique. Il est donc difficile de leur enseigner en anglais des notions dont le référent leur est étranger en français.

8 Nous n'avons pas pour but de leur enseigner l'informatique, et selon les termes de J.-M. Baissus: "la langue de spécialité ne peut pas marcher s'il n'y a pas un acquis professionnel préalable $»^{1}$. Notre tâche est cependant rendue plus complexe par le fait que certains ont choisi une option informatique au lycée de la seconde à la terminale, et aussi du fait de la place de l'informatique dans la société actuelle.

9 Les étudiants eux-mêmes ont une conscience très vive de ces disparités lors des premières semaines à l'IUT et ceux qui n'ont jamais touché une disquette ressentent quelque malaise face aux « bidouilleurs » qui jonglent avec le jargon de base, souvent en anglais, et manipulent les machines avec une dextérité qui les fascine.

10 S'il ne s'agit pour certains que d'une simple familiarité de surface, d'autres ont déjà acquis de réelles connaissances et des compétences solides. Or, chacun sait, qu'il le déplore ou non, que l'anglais et l'informatique entretiennent des liens étroits! Cela a pour effet que ceux qui ont acquis ces connaissances en informatique ont souvent acquis de pair une terminologie anglaise, sans qu'il y ait pour autant une adéquation entre leur niveau d'anglais « général » et leur niveau en anglais de spécialité. Il conviendrait certes d'affiner ce que l'on entend par niveau, par compétence, qui est d'un autre ordre que la simple connaissance d'une terminologie. Disons, sans entrer dans les détails, que ce lien entre informatique et anglais est pour nous, enseignants d'anglais à des étudiants d'informatique, à la fois un atout et une difficulté.

11 Tout d'abord une difficulté parce que, comme je viens d'y faire allusion, la connaissance de termes n'est pas synonyme d'une véritable compétence langagière, mais donne parfois cette illusion à nos étudiants. Par ailleurs, comme la plupart des emprunts, les mots informatiques anglais sont souvent prononcés "à la française ». Ainsi le mot «data ", familier à nos étudiants n'est pas automatiquement reconnu lorsqu'il est prononcé par un présentateur de CBS.

12 Enfin, les regards affichent souvent un certain étonnement lorsque l'on demande la traduction en français d'un terme tel que «buffer ». Certes, le terme «franco-français » existe et les dictionnaires donnent "mémoire-tampon", mais le terme anglais tend à l'emporter dans l'usage de nos collègues enseignant l'informatique tout comme dans la littérature spécialisée.

13 Toutefois cette « invasion » de l'anglais dans l'informatique présente aussi des avantages non négligeables. Ceux-ci peuvent s'exprimer en termes de légitimité institutionnelle et de reconnaissance de l'enseignant d'anglais, aussi bien de la part de ses collègues spécialistes que des étudiants.

14 Ni les uns ni les autres ne sont en position de remettre en cause le bien-fondé d'un enseignement d'anglais. Cela s'exprime aussi en termes de motivation et d'intérêt pour la discipline et permet à des étudiants dont le niveau d'anglais est faible et qui avaient intériorisé cette faiblesse comme irréversible de changer peu à peu leur perception d'euxmêmes, de prendre confiance et de progresser. Il y a là donc une occasion pour des 
étudiants de niveau moyen, voire faible, en anglais, d'arriver à des performances tout à fait honnêtes.

Cette introduction avait pour but de situer le cadre de notre réflexion sur l'utilisation de publicités informatiques. En effet, ce que nous venons de dire rend compte pour partie de certaines difficultés lors du choix des documents comme support de travail sur la langue de spécialité. Ce choix est épineux car il est difficile de trouver des documents dont aussi bien le niveau de langue que le contenu référentiel soient adaptés. Si l'on choisit des articles faisant appel à des concepts, ils risquent d'être jugés trop abstraits, alors qu'à l'autre extrême les notices d'utilisation, très chargées en lexique technique, manquent d'ancrage énonciatif et sont de ce fait peu exploitables en tant que telles. De plus, l'évolution rapide de l'informatique contraint l'enseignant à une mise à jour constante de ses documents. La formule "oh, ça c'est vieux » étant en gros équivalente à «çà a au moins 6 mois »!

Le moment est donc venu de nous interroger sur les traits caractéristiques du document publicitaire. Que propose-t-il qui lui soit propre et qui mérite notre intérêt? Tout d'abord quel corpus avons-nous retenu?

17 Nous l'avons limité aux publicités informatiques publiées dans les magazines informatiques. Nous ne travaillons donc que sur support papier et sans élément sonore. Notre corpus est constitué de publicités pour produits informatiques, de type matériel ou logiciel, recueillies dans des magazines informatiques de moins d'un an, d'origine britannique et américaine. Citons: PC World, PC Magazine, Personal Computer World, Computer World, What Personal Computer?, Computer Shopper, Computer Buyer, Mac User, Lan, et Compute.

Avant d'entrer dans la matérialité du document, disons tout de suite qu'il serait erroné de penser qu'il s'agit d'un document simple. Rappelons à ce sujet une phrase de A. Huxley :

The advertisement is one of the most interesting and difficult of modern literary

forms. (cité par H. Greven [1982 : 28])

Ce qui a particulièrement retenu notre attention dans la publicité en général et dans la publicité informatique en particulier, c'est son caractère hybride. Elle possède en effet un statut culturel particulier, et même si elle fait référence à un domaine de connaissance scientifique et technique, ce n'est pas d'abord à cette aune-là qu'elle est jugée. Une publicité, en raison de son caractère polymorphe, suppose la maitrise de plusieurs moyens de communication et d'expression, disons pour faire vite, des moyens picturaux et discursifs puisque notre corpus a écarté l'élément sonore.

Reprenons la distinction proposée par J.-M. Baïssus dans les Cahiers de l'ILSER (Baïssus 1984 : 27) où il explique sur un schéma à deux axes que la langue scientifique se déplace sur l'axe dénotatif, selon son degré de non-ambiguïté, alors que la poésie se situe à l'extrême de l'axe connotatif. Or, les publicités ont pour caractéristique de base de jouer à des degrés divers sur les deux registres: le registre connotatif et le registre dénotatif. Nous verrons que les publicités informatiques que nous avons étudiées privilégient l'aspect dénotatif, à visée informative. Elles se démarquent ainsi de publicités qui jouent exclusivement sur l'évocation suggestive, mais ne donnent aucune information, telle cette affiche publicitaire pour une voiture :

Elle plaît, c'est son seul défaut

21 Certes, l'objectif recherché par les publicités informatiques est identique. Il s'agit dans un cas comme dans l'autre de convaincre un acheteur et les moyens de convaincre sont 
similaires : faire l'éloge du produit. Ce qui varie, ce sont les éléments sur lesquels se fonde cet éloge. Ce que nous avions remarqué en parcourant des publicités informatiques, c'était la part, certes variable, mais toujours non négligeable de texte, ce qui n'est pas le cas de toutes les publicités. En ce qui concerne notre corpus, c'est l'aspect technique du produit qui sert d'ancrage et de fondement à la valeur du produit, et c'est en bonne part pour cette raison qu'elles nous semblent être un support adaptable à notre enseignement de l'anglais de l'informatique. Nous verrons plus loin quelles activités nous avons proposées à nos étudiants à partir de ces publicités, mais une autre caractéristique de toute publicité nous paraît valoir la peine d'être rappelée, c'est son caractère " accrocheur », qui n'est pas nécessairement négatif.

Reprenons à ce propos les buts de la publicité tels que les a définis H. Greven (1982). Il s'agit en particulier d'attirer l'attention, d'éveiller et maintenir l'intérêt, d'exhorter, enjoindre et de vaincre la passivité du destinataire.

Ces objectifs ne sont-ils pas aussi des objectifs didactiques, si on les dépouille de leur fin commerciale ? Ajoutons à cela que le concepteur de publicité cherche à faire en sorte que son destinataire garde en mémoire quelque chose de ce qu'il a lu ou vu, ou entendu. C'est aussi ce que nous espérons, en tant qu'enseignants, même si le contenu que nous souhaitons faire mémoriser n'est pas du même ordre.

Il n'en reste pas moins vrai que pour parvenir à ces objectifs, le concepteur de publicité met en œuvre des moyens spécifiques. Ce sont ces moyens auxquels nous allons nous intéresser, tout d'abord sur le plan général de la communication publicitaire et dans un deuxième temps, plus spécifiquement, en ce qui concerne notre objet.

Entrons donc plus avant dans la matérialité du document publicitaire. Comme tout document, une publicité est une mise en scène, au sens théâtral du terme. Si cela est vrai de n'importe quel document - on sait l'importance d'une mise en page - cela est particulièrement déterminant dans le domaine publicitaire. Il s'agit là d'une véritable architecture qui structure l'espace, définit des rapports entre le ou les textes et le ou les éléments iconiques afin de capter l'attention du destinataire. Car destinataire il y a, explicitement ou implicitement présent dans le message, de même qu'il y a énonciateur, implicite ou explicite (les traces les plus évidentes étant la présence de « we » ou de « you " qui donnent au lecteur l'illusion d'un contact personnel). Il s'agit donc d'une langue en fonctionnement. C'est la raison pour laquelle on y trouve l'essentiel des niveaux de langue tels que M. Perrin les modélise sous forme pyramidale (1992).

La publicité construit donc un univers structuré autour d'un point fort: le produit, ou plus exactement une image de ce produit. Il s'agit donc d'un document focalisé, concentré, condensé, qui présente une forte cohérence et ces éléments sont précieux en matière de didactique. Par ailleurs, même si notre objectif est un objectif linguistique, à savoir faire acquérir une meilleure compétence de l'anglais de spécialité et de l'anglais général, nous n'oublions pas que cette acquisition se fait selon des itinéraires qui varient selon les personnes, selon les contenus, voire selon les moments, etc. Sur ces itinéraires, dont on peut penser qu'ils ne sont pas linéaires, certains éléments peuvent fonctionner comme catalyseurs et médiateurs de sens.

27 Parmi ceux-ci, l'image joue un rôle que l'on ne saurait minimiser. Non pas que l'image soit nécessairement plus simple à comprendre, si l'on se réfère par exemple aux travaux de $\mathrm{F}$. Bresson (1981) sur la lecture de l'image par comparaison à la lecture d'un texte. 
28 Toutefois l'image peut être appréhendée dans sa globalité alors que le texte est nécessairement linéaire. Bien entendu le texte peut aussi fonctionner comme un élément iconique et être d'abord perçu comme tel grâce aux multiples jeux de la typographie. C'est un aspect dont l'art publicitaire joue avec talent.

Cet art du jeu est vraisemblablement un des éléments-clés de la publicité, dans ses multiples acceptions. Par jeu il s'agit d'entendre aspect ludique mais aussi jeu au sens de «laisser du jeu », c'est-à-dire une certaine forme de liberté. Ainsi, la publicité joue sur plusieurs tableaux, elle est polymorphe, elle peut être appréhendée sous plusieurs angles. On y trouve jeu sur les formes, jeu sur les mots, jeu sur le sens, et jeu sur les sens (visuel, sonore...). Elle fait appel au rationnel et à l'imaginaire.

Pour cette raison, même si elle contient des éléments de langue technique et scientifique, elle ne saurait être considérée comme de la langue scientifique. L. Trimble (1985) définit en effet la langue scientifique comme la transmission d'informations sans utilisations des fonctions rhétoriques telles que l'argumentation non logique, les images poétiques, la création d'émotions, du rire. Or, ces éléments sont justement des éléments constitutifs du fonctionnement publicitaire. Comment apparaissent-ils dans notre corpus, comment s'articulent-ils avec les éléments purement référentiels présentés et qui forment dans la majorité des cas le noyau de nos publicités?

31 Nous avons dit plus haut que notre intérêt didactique pour la publicité informatique avait été éveillé par la constatation qu'elles offraient au lecteur une proportion non négligeable de texte, prenant même parfois la forme de "petit roman » sur plusieurs pages. Or le texte a dans l'image une fonction d'ancrage du sens, selon les termes de R. Barthes (cité par Cornu 1990). Il dépasse le cadre de notre propos d'analyser les rapports entre l'image et le texte dans les publicités, disons simplement qu'une tendance se dégage nettement: l'argumentaire publicitaire informatique, s'il ne néglige pas l'image, double celle-ci d'un contenu informatif référentiel.

Certes, il existe des styles différents, des éclairages et des mises en perspective variables selon les magazines, chacun ayant sa propre marque, qui peut dépendre du type de public visé, également du type de produit présenté. Par exemple, il est facile d'avoir recours à un mode de représentation physique si l'on fait l'éloge d'un ordinateur, c'est plus difficile lorsqu'il s'agit de produits logiciels. Il n'est donc pas étonnant que ceux-ci donnent davantage naissance à des représentations symboliques. Il s'agit là de contraintes liées à la nature même du produit vanté. N'en concluons pas pour autant que c'est la nature du produit qui détermine la nature de la publicité.

33 En effet, le fonctionnement publicitaire de base repose sur l'exaltation du produit que l'on cherche à vendre, en en vantant les vertus, par un système de valeur ajoutée. Ce n'est pas tant un produit que l'on achète, mais des valeurs qui lui sont attachées. Un fabricant de chaussures ne disait-il pas «Je ne vends pas des chaussures, je vends des jolis pieds». Or, ces valeurs ajoutées peuvent être de plusieurs types.

34 Il n'est pas possible de développer ici cet aspect du fonctionnement publicitaire, mais il existe, là comme ailleurs, des écoles de pensée qui s'affrontent et d'autre part, la publicité, liée comme elle l'est à la culture, est soumise aux différences de lieux et d'époques.

Il serait sans nul doute très instructif d'étudier comment un même produit est mis en scène différemment selon le public-cible visé et comment les éclairages auxquels il est soumis évoluent dans le temps. Dans un domaine à évolution aussi rapide que 
l'informatique, on y trouverait matière à réflexion sur la place et l'image de l'informatique à travers le temps et les cultures.

Notre propos sera beaucoup plus modeste et nous ne prétendons nullement offrir un panorama exhaustif des types de publicités informatiques. Essayons cependant de dégager des tendances à partir de notre corpus et en gardant à l'esprit notre objectif de base : prendre appui sur ces publicités pour amorcer un travail en langue anglaise et plus particulièrement en anglais de spécialité informatique.

Prenons un exemple révélateur d'un certain type de valorisation publicitaire. Une affiche publicitaire actuellement sur nos murs se résume à ceci : l'image d'une voiture, son nom, un visage aux yeux bandés, et ce slogan :

j'achète les yeux fermés

Il s'agit de ce que l'on peut appeler une publicité de marque, la valeur tenant tout entière dans une référence à la marque, dont le nom à lui seul suffit à cristalliser tout un éventail de valeurs qu'il est inutile d'expliciter puisque le nom de la marque est identifié à ces valeurs que l'on pourrait résumer d'un mot : l'excellence ! Rien n'est dit dans cet exemple des qualités techniques du produit à l'affiche.

Si la voiture nous permet une comparaison intéressante, c'est parce qu'il s'agit d'un produit hautement technologique lui aussi et que ce n'est donc pas la nature du produit qui suffit à justifier les choix d'argumentation publicitaire. Certes, ce n'est qu'un exemple parmi d'autres et il s'agit d'une affiche, donc d'un support différent, source de contraintes différentes.

Il n'en reste pas moins vrai qu'actuellement la plupart des publicités informatiques utilisent un argumentaire opposé, et cherchent à convaincre en conviant l'acheteur potentiel non pas à acheter les yeux fermés, mais au contraire à les avoir grand ouverts, afin d'acheter en « connaissance ». C'est ainsi que l'on vous fait découvrir un ordinateur sous des angles différents, voire qu'on le désosse et qu'on en exalte les vertus de façon explicite, avec preuves à l'appui.

Quelle est la nature de ces preuves et quelles valeurs exaltent-elles?

L'éloge publicitaire consiste à placer un produit sur une échelle de valeurs. Ces valeurs sont, pour ce qui nous concerne, des valeurs de performance, efficacité, simplicité, fiabilité, rapidité, haute technicité et innovation.

Pour ne donner que les principales. La technique rhétorique consiste donc non pas tant, ou du moins pas seulement, à égrener ces valeurs à la façon d'une litanie, mais à convoquer des arguments comme témoins de ces valeurs. Ces arguments-preuves sont pour une bonne part une explicitation et une mise en valeur des caractéristiques techniques du produit.

Cela ne tient pas tant, encore une fois, au produit lui-même qu'au destinataire et à la représentation qu'il se fait de ses besoins et du rôle que peut jouer l'informatique pour lui. Cela participe aussi de la fonction mythique remplie actuellement par la haute technologie et l'informatique dans notre culture.

Il s'agit donc, pour reprendre la terminologie de Jean-Marie Floch (1990) d'une publicité de type référentielle, c'est-à-dire centrée sur le produit dans sa réalité matérielle. $\mathrm{Ce}$ choix est lié à l'image que l'on souhaite construire dans un lieu et à un moment donné parce que c'est cette image qui est efficace commercialement.

Quelle est donc cette image lorsqu'il s'agit des publicités informatiques? 
47 Nous avons déjà dit qu'elles contenaient de l'information, mais voyons comment celle-ci est structurée et de quel type d'information il s'agit. Reprenons les catégories proposées par J.-M. Floch (op. cit.). Celui-ci considère que: «deux grands régimes partagent la création publicitaire : l'un relève de la dénotation référentielle, l'autre de la connotation à visée psychologique ». Même si ces deux éclairages peuvent coexister dans une même publicité, il apparaît que les publicités qui nous occupent ressortissent essentiellement du premier type.

Quels sont les traits dominants de la publicité référentielle ? Son objectif est de tendre au réalisme, c'est-à-dire non pas d'être vrai, mais de faire paraître vrai et les moyens utilisés pour atteindre cet objectif sont : e recours à des photos de préférence aux dessins. Comme l'indique H. Greven : « les photographies, naturelles et réalistes, sont donc apparemment dignes de confiance », mais « les dessins offrent l'avantage de styliser et d'idéaliser ». Il ajoute que les retouches sur photos permettent de jouer sur les deux tableaux.

Au niveau discursif, et je cite à nouveau J.-M. Floch, la publicité référentielle repose sur des discours : narratifs, figuratifs (et non abstraits) et descriptifs (et non normatifs).

En ce qui concerne le système de valorisation, J.-M. Floch distingue quatre types de valorisation possibles d'un produit, l'une n'excluant pas nécessairement les autres. Il s'agit de : valorisation pratique (valeurs d'usage, utilitaires, fonctionnelles), valorisation utopique (identité-vie-aventure), valorisation ludique (luxe-gratuité-raffinement) et valorisation critique (qualité-prix/innovation-coût).

51 Le travail que nous avons mené nous permet de dire que c'est surtout le premier type de valorisation qui est privilégié, ainsi que le dernier. La communication orale faite à Montpellier s'appuyait pour une grande part sur une "promenade» guidée et commentée à travers une vingtaine de publicités représentatives des modes de fonctionnement. Dans les limites de cet article, il n'est malheureusement pas possible de reproduire l'ensemble du dossier distribué aux participants. Que nos lecteurs nous en excusent. Nous nous limiterons à regret à commenter deux exemples qui ont servi d'amorce au travail avec les étudiants, mais qui ne donnent qu'une image partielle et partiale d'un corpus aux multiples facettes.

Nous donnerons ensuite les différentes activités que nous avons mises en place. Nous terminerons par une synthèse de la discussion qui a suivi la communication et dont la trace écrite ne saura rendre la richesse.

La première publicité (voir figure 1) que nous commenterons s'intitule: "We just rewrote the notebook». Il s'agit d'une publicité pour un type de portable, le notebook de Toshiba dont l'original se déployait sur deux grandes pages dans le numéro de P.C. World de décembre 1991. La réduction et la perte des couleurs, bien que celles-ci aient été dans ce cas précis, limitées à quelques nuances de bleu et de gris, appauvrissent considérablement la qualité du document, mais qu'y faire?

Figure 1 Publicité « We just rewrote the notebook » 


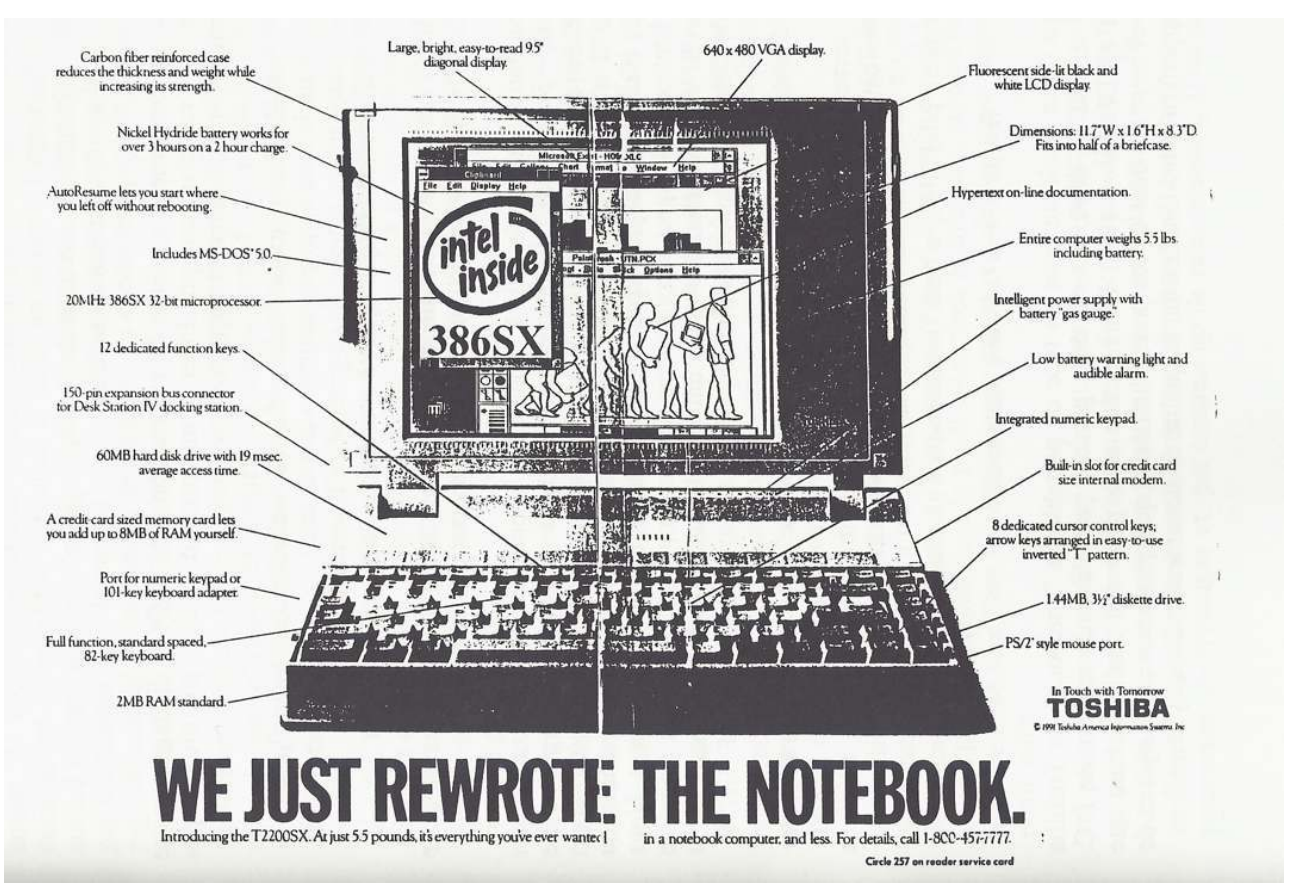

La mise en page, qui ne cherche guère d'autres effets que celui du réalisme, est typique d'une focalisation sur le produit. Celui-ci occupe une position centrale et est auréolé de ses caractéristiques techniques grâce au faisceau de flèches dont le rayonnement symbolique sert à orienter le regard du destinataire vers autant de cibles, constituées essentiellement par les spécifications de l'ordinateur. L'ensemble contribue à créer un univers réaliste, technique, auquel s'ajoute un souci "pédagogique » de désignation et d'étiquetage, illustré par les flèches, qui donnent à ce document une certaine similarité avec une page de manuel ou une notice de montage, ou encore une page de dictionnaire encyclopédique du type What's What.

Le lecteur est ainsi guidé, orienté vers ce qu'il convient qu'il remarque, vers ce qui fait la valeur de l'objet. Il s'agit bien d'une publicité de type référentiel, avec prédominance de la représentation physique et le souci du détail fonctionnel.

Ceci dit, la représentation d'un ordinateur fournit une possibilité toute trouvée d'images dans l'image, grâce à l'écran qui fonctionne à la fois comme contenu à montrer et comme support pour montrer. Il permet ainsi un affichage au second degré, voire davantage, grâce au multifenêtrage dont on a ici un exemple probant.

57 Ici, le triple fenêtrage permet de visualiser l'affichage à l'écran avec le commentaire "large, bright easy-to-read 9.5 " "diagonal display ", ce qui sert à la fois à donner des informations techniques, mais constitue bien évidemment un argumentaire sous-jacent: «il est petit certes, mais l'écran, lui, ne l'est pas ».

Une seconde fenêtre permet d'indiquer le type de microprocesseur, ici un INTEL 386, élément interne et donc non représentable sur cette image, mais information capitale pour un utilisateur averti puisqu'il conditionne la puissance de la machine. Les polices de caractères utilisées dans cette fenêtre mettent en relief l'importance donnée à cette information.

59 La troisième fenêtre se situe sur un tout autre registre, de type symbolique, cette fois. S'y déploie un raccourci de l'évolution de l'homme et, en parallèle, de l'évolution de l'ordinateur. L'argumentaire étant schématiquement: par le passé l'homme ployait sous 
le poids d'énormes ordinateurs, aujourd'hui il se redresse et se sent libre de ses mouvements grâce à des appareils légers et de petite taille.

On peut voir assez facilement quel parti tirer de cette publicité pour une étude du vocabulaire de base référant au matériel informatique. Il s'agit en fait d'un catalogue de termes techniques, de type nomenclature, mais fléchée et structurée visuellement, ce qui ne saurait être négligeable en termes de perception, de repérages et, par contrecoup de mémorisation. D'un point de vue linguistique cet inventaire est particulièrement riche en substantifs tels que : bus connector, memory card, keypad, mouse port.

Comme il est fréquent en informatique, nombre d'entre eux sont des substantifs composés et « l'enfilage » de déterminants appartenant à des catégories multiples (noms, adjectifs simples et composés, mais aussi chiffres et sigles) y est très développé. Tout enseignant sait à quel point cette « antéposition multiple » est difficile à maitriser pour un Français.

Cette page en offre quelques exemples:

150-pin expansion bus connector

a credit-card sized memory card

fluorescent side-lit black and white LCD display

full function, standard spaced, 82-key keyboard.

Il y a là tout un potentiel linguistique à exploiter, en compréhension et en production. $\mathrm{Ne}$ peut-on par exemple imaginer de garder l'image et les flèches et de donner les termes sous forme de "scrambled expressions" ou de "puzzle» à reconstituer. Ceci pouvant ensuite donner lieu à un travail de généralisation et de réflexion sur la détermination, ancré dans des exemples maîtrisés puis prolongé par-delà les exemples ici présents et dont l'informatique offre un éventail ample et varié.

Si cette page est riche en substantifs et en déterminants, elle l'est par contre, fort peu en verbes. Il y a là cohérence avec l'éclairage choisi : mise en relief des caractéristiques physiques plus que des fonctions. L'essentiel du texte est non-narratif et ne présente que peu de phrases construites. C'est là une de ses limites. Par ailleurs, et cela est également cohérent avec le souci du "faire paraître objectif », la prise en charge énonciative est quasi inexistante, donnant à l'ensemble un aspect de neutralité objective qui constitue en l'occurrence un choix argumentatif. Les traces de la présence de l'énonciateur sont discrètes et limitées à « we » dans le slogan : « we just rewrote the notebook », qui représente une condensation de l'argumentaire. Ce terme «just » apparaît de façon récurrente dans notre corpus, la simplicité faisant partie des arguments de base.

65 À en juger par la réaction de mes étudiants, que ce slogan a laissé perplexe, il ne me paraît pas inutile de le donner à « décondenser » afin d'en faire apparaitre de façon non ambiguë la logique sous-jacente. Cette tâche aurait pour intérêt, outre l'effort d'explicitation, de donner aux étudiants l'occasion de sortir du cadre strict de la référenciation ou de l'étiquetage et d'articuler une pensée, d'avoir recours à des connecteurs logiques, bref, d'argumenter eux-mêmes.

La seconde publicité dont j'ai choisi de faire une exploitation collective avec les étudiants s'intitule: "What kind of computer does it take to sell 5000 a day? " (voir figure 2).

Figure 2 Publicité « WHAT KIND OF COMPUTER dOES IT TAKE TO SELL 5000 A DAY? » 


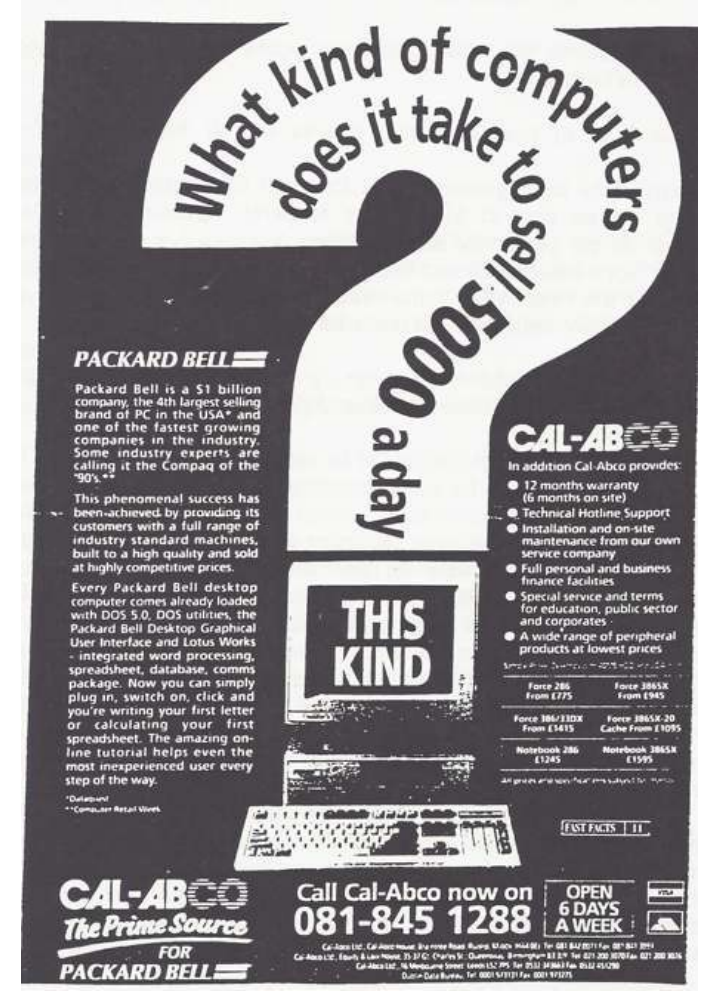

Elle a paru dans le magazine What Personal Computer de janvier 1992. L'original est en noir et blanc donc la perte visuelle est faible sur la photocopie, ce qui a constitué un des critères de choix. Les étudiants travaillent ensuite de façon individuelle sur des originaux et leur choix a confirmé le rôle prédominant du visuel dans leurs critères de sélection. Toutefois, pour un travail préliminaire collectif cette publicité a ses atouts.

Contrairement à la précédente, l'image s'y veut à la charnière du figuratif et du symbolique, le figuratif étant au second plan et peu détaillé. L'accroche joue sur la typographie et la forme, elle se moule dans l'énorme point d'interrogation qui à lui seul structure presque toute la moitié supérieure de la page. Alors que la précédente laissait peu de place au destinataire et à l'énonciateur, celle-ci commence par créer un lien entre les deux partenaires en lui posant une question, celle de l'accroche à laquelle il s'empresse bien sûr de répondre à sa place : «this one ». Il y a donc appel au lecteur que l'on rend témoin de la réussite de la firme en question. L'argumentaire est ici centré explicitement sur la notion de succès, succès dont on peut inférer que le produit est de qualité. La partie iconique sert de séparation entre deux textes de type et de fonction différents.

Le texte de gauche est un texte narratif, explicatif qui s'adresse à " vous, utilisateurs ». Le texte de droite présente des informations brutes, en style télégraphique, de type plus commercial que technique (service après-vente, garantie, éventail des prix...). Ce type de vocabulaire qui n'est que peu ou pas familier à nos étudiants fait partie de la langue de communication professionnelle et il est bon qu'ils la maîtrisent.

Pour ce qui est du vocabulaire informatique à proprement parler, on y trouve les substantifs suivants : word processing, spreadsheet, database et une forme d'abréviation qui peut poser problème : « comms package » pour communications package.

71 Le ciblage vers l'utilisateur s'exprime par des verbes tels que : to plug in, to switch on, to click, to calculate. 

repérer. Une étudiante ne me disait-elle pas à propos d'un travail sur les virus: «en français, on dit anti-virus, mais en anglais ça ne doit pas être pareil ». Si, c'est " pareil », mais cela n'est évident que pour celui qui a rencontré la forme anglaise. Au niveau syntaxique, on trouve des passifs, mais aussi des superlatifs :

the 4th largest selling brand of PC in the USA.

one of the fastest growing companies.

74 Ces deux exemples présentent une structure qui n'est pas exactement celle qui leur est familière et la traduction n'est pas sans intérêt. Cette publicité ne comporte pas de comparatifs bien que de façon générale, les publicités soient un terrain favorable à la prolifération des degrés de comparaison.

Nous allons revenir sur les applications pédagogiques dont j'ai déjà dit un mot au fil de ces commentaires mais avant de quitter les publicités elles-mêmes et pour donner un aperçu de leur richesse, voici quelques exemples de jeux sur la langue que j'ai pu y glaner.

Tea for Two

représente deux tasses à thé contenant des cartes d'interface nommées 10 Base-T, d'où la conclusion : this is your cup of $\underline{T}$.

A Cure for the Terminal Blues

avec jeu sur le double sens de Terminal.

The Great British Drive

qui joue sur les deux sens de drive en représentant une superbe voiture ancienne et un disk drive.

Now Ross uses Digital's NAS to open Business Ties

la publicité étant un réseau.

The new HP PCs : From not working to networking qui joue sur l'allitération et le contraste phonique.

77 Terminons par cette délicieuse trouvaille pour un anti-virus qui montre un groupe de lièvres, toutes oreilles dressées, avec cette accroche :

Finding a virus can be a Hare-Raising experience.

78 Au cas où la raison d'être du choix de " hare », au-delà de l'homophonie « hair » ne serait pas évidente au lecteur, la cohérence entre l'image et le jeu de mots est explicitée par :

They multiply, multiply, multiply!

Aussi savoureuses ou insolites qu'ils puissent nous paraître, ces jeux sur la langue sont le plus souvent obscurs à nos étudiants, et nécessitent une explicitation qui s'est révélée une excellente occasion de travailler sur l'analogie, la comparaison et le contraste, autant de techniques rhétoriques spécifiques de la langue scientifique, si l'on se réfère à $\mathrm{L}$. Trimble.

Ils sont aussi le point de départ d'un travail lexical, le jeu didactique pouvant consister à donner uniquement l'accroche qui contient un jeu sur des mots et à faire deviner le produit informatique, l'image suggestive, et la cohérence sous-jacente.

81 Quant aux icônes, la gamme en est variée, elle aussi, et si la représentation l'emporte, d'autres registres y ont aussi leur place, du comique à l'insolite en passant par le romantique, l'écologique, l'esthétique, etc. Les animaux, les enfants, les sportifs apparaissent comme support à l'argumentation, ainsi que les hommes d'affaires, mais l'image de la femme y est encore assez peu fréquente, bien que l'on puisse percevoir une évolution. 
82 Essayons maintenant de faire un bref tour d'horizon des applications didactiques envisageables au niveau du DUT Informatique, c'est-à-dire, pour des étudiants se préparant à devenir des techniciens. Les publicités peuvent servir de supports à des activités de compréhension écrite, production écrite et production orale.

Un premier travail a consisté en un travail de compréhension et de classement. Nous nous sommes inspirée de la technique préconisée par L. Trimble. Celle-ci consiste à structurer le travail de repérage en plusieurs étapes, au cours desquelles le guidage de l'enseignant s'estompe, puis disparaît.

Dans un premier temps, la même publicité est distribuée aux étudiants ainsi qu'une grille de repérage reproduite ci-après (voir figure 1).

Figure 3

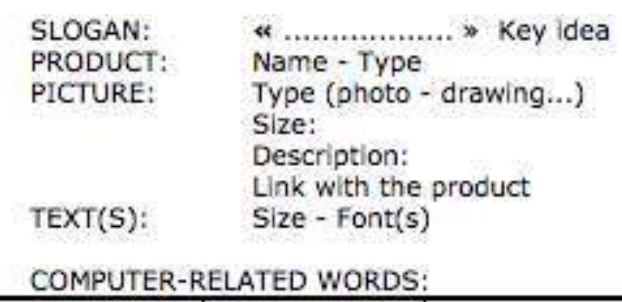

\begin{tabular}{|l|l|l|l|l|}
\hline nouns & verbs & adjectives & adverbs & expressions \\
\hline & & & & \\
\hline
\end{tabular}

TECHNICAL WORDS:

\begin{tabular}{|l|l|l|l|l|}
\hline nouns & verbs & adjectives & adverbs & expressions \\
\hline & & & & \\
\hline
\end{tabular}

QUALITIES OF THE PRODUCT:

\begin{tabular}{|l|l|l|l|l|}
\hline nouns & verbs & adjectives & adverbs & expressions \\
\hline & & & & \\
\hline
\end{tabular}

KEY ARGUMENTS:

PEOPLE MENTIONED:

TERMS OF SALE:

Il s'agit par ce travail de faire prendre conscience de la structure de la publicité, en tant qu'image et que texte, de l'argumentaire mis en œuvre et d'analyser les moyens qui ont servi de matériaux de construction à cet argumentaire. Ce travail de repérage, de tri et de classement au niveau iconique et textuel suppose une lecture attentive et critique, une perception à la fois globale, linéaire et structurée, ce qui est en soi un acte de compréhension, mais aussi déjà d'acquisition.

86 Cette grille a pour but de guider les étudiants et d'aider dans une tâche de catégorisation ceux d'entre eux qui ont le plus besoin de structurer leur pensée.

87 À l'issue de cette étape, ils sont invités à confronter leur butin avec celui de leur voisin ou voisine. Cette technique me paraît plus fructueuse qu'un travail commencé en binôme car elle oblige chacun à s'interroger pour lui-même et à prendre, dans un premier temps, le risque de ses propres réponses tout en permettant, grâce à l'interaction qui suit, de procéder à des vérifications et des ajustements entre pairs, avant d'affronter le groupe dans l'étape d'oralisation qui suit. 

description fonctionnelle, la classification, les instructions, les relations verbalesvisuelles. Je renvoie ici encore aux catégories mises en évidence dans ce domaine par L. Trimble (1985). multiples prolongements, si l'on $\mathrm{y}$ trouve de la langue technique très pointue compréhensible aux seuls initiés (en général moins ésotérique pour nos étudiants que pour nous-mêmes), mais aussi de la langue de communication, tout comme des recherches d'effets de mots et de sens, en revanche il ne s'y trouve guère, et cela se conçoit, de langue abstraite, renvoyant à des concepts. 
un des atouts, et il est précieux dans un domaine où l'évolution est constante et rapide, la mise à jour des documents est facile et permet une souplesse d'utilisation appréciable. Les étudiants y sont sensibles et apprécient notre désir d'adaptation et d'adéquation à leur univers de travail.

\section{BIBLIOGRAPHIE}

Baissus. J. M. 1984. « Pour une pédagogie du sens ». Cahiers de l'Ilser 3.

Bresson, F. 1981. «Compétence iconique et compétence linguistique ». Communications 33, 185-195.

Cathelat, B. \& R. Ebguy. 1988. Styles de pub, 60 manières de communiquer. Paris : Éditions d'organisation.

Cornu. G. 1990. Sémiologie de l'image dans la publicité. Paris : Éditions d'organisation.

Floch, J. M. 1990. Sémiologie, marketing et communication. Sous les signes, les stratégies. Paris : Presses Universitaires de France.

Greven, H. 1982. La langue des slogans publicitaires en anglais contemporain. Paris : Presses Universitaires de France.

Perrin, M. 1992. « De l'utilisation communicative des documents authentiques ». In Perrin, M. (dir.), Actes du XI Colloque du Geras. Bordeaux : GERAS, 9-32.

Trimble, L. 1985. English for Science and Technology, A discourse approach. Cambridge : Cambridge University Press.

\section{ANNEXES}

\section{Annexe : Compte-rendu des discussions}

La discussion qui a suivi a fait émerger les remarques suivantes.

L'univers technique construit par ces publicités n'est-il pas un leurre, visant à noyer l'acheteur potentiel sous un déluge de jargon technique auquel il ne comprend rien. À cette interrogation, il a été répondu que tout dépendait du destinataire, et fait remarquer que les publicités pour grand public étaient moins chargées de ce point de vue, qu'il s'agissait dans ce corpus de publicités tirées de magazines spécialisés et qu'en conséquence leurs lecteurs fondaient leurs choix sur des spécifications techniques, lesquelles fonctionnaient alors comme argument de vente.

Une suggestion a été faite concernant l'utilisation des publicités parallèles en français. Il y a là en effet un potentiel auquel il convient de réfléchir. Ce qui apparaît de façon massive, c'est que même lorsque l'on trouve pour le même produit des publicités identiques quant à l'image, le texte est complètement remanié. Il y a là manifestement une piste à exploiter en vue d'une analyse contrastive culturelle et linguistique. 
Enfin il a été fait remarquer que l'évolution des publicités pour un même produit, pour une même marque, ou pour un même type de produit pourrait ouvrir des perspectives intéressantes, et révéler quelque chose de l'évolution de l'informatique et de sa place dans nos sociétés.

Cette dernière remarque, en prenant en compte le facteur temporel inclut par là même une dimension culturelle et rappelle, s'il en était besoin, que technologie et culture sont interdépendantes. S'intéresser à l'univers technologique ne signifie donc pas pour autant que l'on se désintéresse de l'univers culturel qui est le nôtre.

\section{NOTES}

1. Communication faite lors de la session initiale du DEA de langue anglaise des spécialités scientifiques et techniques.

\section{AUTEUR}

\section{THÉRĖSE BROUAT}

Département Informatique, IUT de Montpellier.thbmartin@yahoo.fr 